\title{
Design of FIR Paraunitary Filter Banks for Subband Coding Using a Polynomial Eigenvalue Decomposition
}

\author{
Soydan Redif Member, IEEE, John G. McWhirter and Stephan Weiss, Senior Member, IEEE
}

\begin{abstract}
The problem of paraunitary filter bank design for subband coding has received considerable attention in recent years, not least because of the energy preserving property of this class of filter banks. In this paper, we consider the design of signal-adapted, finite impulse response (FIR), paraunitary filter banks using polynomial matrix EVD (PEVD) techniques. Modifications are proposed to an iterative, time-domain PEVD method, known as the sequential best rotation (SBR2) algorithm, which enables its effective application to the problem of FIR orthonormal filter bank design for efficient subband coding. By choosing an optimisation scheme that maximises the coding gain at each stage of the algorithm, it is shown that the resulting filter bank behaves more and more like the infiniteorder principle component filter bank (PCFB). The proposed method is compared to state-of-the-art techniques, namely the iterative greedy algorithm (IGA), the approximate EVD (AEVD), standard SBR2 and a fast algorithm for FIR compaction filter design, called the window method (WM). We demonstrate that for the calculation of the subband coder, the WM approach offers a low-cost alternative at lower coding gains, while at moderate to high complexity, the proposed approach outperforms the benchmarkers. In terms of run-time complexity, AEVD performs well at low orders, while the proposed algorithm offers a better coding gain than the benchmarkers at moderate to high filter order for a number of simulation scenarios.
\end{abstract}

Index Terms-Orthonormal subband coders, paraunitary matrix, principal component filter banks, polynomial matrix eigenvalue decomposition, sequential best rotation.

\section{INTRODUCTION}

$\mathbf{P}$ ARAUNITARY filter banks have been extensively studied for subband coding and applied to an increasing number of applications, including noise reduction [1], audio and image coding [2] and digital communications [3], [4]. For the case where the order of the filters is unconstrained, it is known that a principal component filter bank (PCFB) [5], [6] exists and is an orthonormal or paraunitary (PU) filter bank that is simultaneously optimal for a number of objectives [7], including mean-squared error and coding gain for subband coding in data compression applications [8]. This is also true when the filter orders are constrained to be not greater

Copyright (c) 2011 IEEE. Personal use of this material is permitted. However, permission to use this material for any other purposes must be obtained from the IEEE by sending a request to pubs-permissions@ieee.org.

S. Redif is with the Electrical and Electronic Engineering Department, European University of Lefke, Lefke, Cyprus (e-mail sredif@eul.edu.tr).

J.G. McWhirter is with Cardiff University, Cardiff CF24 3AA, Wales, UK (e-mail mcwhirter@cardiff.ac.uk)

S. Weiss is with the Department of Electronic \& Electrical Engineering, University of Strathclyde, Glasgow G1 1XW, Scotland, UK (e-mail stephan@eee.strath.ac.uk) than the number of subband channels. In this case, the zeroorder PCFB, viz. the Karhunen-Loeve transform (KLT) or the singular-value decomposition (SVD) [10], provides the optimal solution [11]. The PCFB also exists for the special case of the two-channel filter bank. However, it is believed that the PCFB does not generally exist for the case where order-constrained (or finite McMillan degree [12]) filters are used [7], [13].

A number of authors have proposed methods for the design of suboptimal finite impulse response (FIR) PU filter banks. Typically, the filter is chosen to optimise a specific objective function for a known input power spectral density (PSD), such as coding gain [2], [14], [15] and multiresolution energy compaction [16]. As a consequence, all such methods require the numerical optimisation of nonlinear and non-convex functions. A common approach has been to calculate an optimal FIR compaction filter for the first filter [17], [18], and then find the remaining filters using an appropriate completion strategy to construct the filter bank [16], [19]. In [16], the FIR compaction filter design problem is reduced to a semi-infinite linear (SIP) program. The authors solve a discretised version of the SIP using standard linear programming methods, which becomes computationally costly and complex for large filter orders. A more efficient discretisation method is proposed in [17], called the window method. However, the main disadvantage of this type of approach is that global optimality is not guaranteed due to the fact that there is ambiguity caused by the nonuniqueness of the FIR compaction filter [18], [19]. In [20], Tkacenko and Vaidyanathan propose a different strategy for the design of filter banks, called the iterative greedy algorithm (IGA), which involves simultaneously designing all of the filters at once, thus avoiding the need to compare the performance of different spectral factors of a given FIR compaction filter. The IGA parameterises a dyadic-based structure, similar to that in [12], by minimising the difference between a desired response and a causal FIR PU filter bank consisting of degree-one paraunitary building blocks. A drawback of this algorithm is that it is very demanding computationally.

PU filter bank design has also been presented in the context of the eigenvalue decomposition (EVD) of para-Hermitian matrices in [22], [23], [24] and signal subspace analysis of broadband signals [25], [26], [27]. The approach by Regalia and Loubaton [25] exploits the fixed degree parameterisation proposed in [12]. They re-formulate the problem using a state space model and propose an iterative solution, which avoids the problems of local minima associated with gradient descent 
techniques. Lambert et al. [27] have developed an EVD for polynomial matrices by generalising some conventional linear algebra and control technique from the complex number field to the field of rational functions. Requiring the approximate inversion of FIR filters in the frequency domain, issues around stability arise.

An alternative PU filter bank design can be obtained by generalisation of the EVD to polynomial matrices, such as the second order sequential best rotation (SBR2) algorithm, proposed by McWhirter et al. in [22]. This algorithm has been successfully applied to broadband extensions of narrowband problems, which traditionally have been addressed by the EVD, including subspace decomposition. In constrast with IGA, where a fixed constraint is imposed on the order of the filters, the filter order of the filter banks constructed by SBR2 can grow with every iteration.

Tkacenko [24] has recently proposed a fixed order algorithm for approximating the polynomial matrix EVD based on applying a given number of degree-one (second order) filter stages. Like the SBR2 algorithm, it aims to increase the zero order diagonal energy in a monotonic fashion. However, this method, referred to as the approximate EVD (AEVD) algorithm, is not designed (or claimed) to converge as the number of stages increases and its performance has been found to saturate at a much lower level than that of SBR2. While a detailed discussion is beyond the scope of this paper, the AEVD is included as one of several benchmarkers below.

In this paper, we consider the application of the SBR2 algorithm to the problem of PU filter bank design for subband coding. A new cost function is proposed, which is based on the coding gain, and improves the diagonalisation and coding gain performances compared to the SBR2 algorithm, for which initial results have been reported in [28]. The resultant algorithm can converge to a solution that yields a PU filter bank, which is approximately optimal for subband coding, in a relatively small number of iterations. It is shown that the resulting filter banks tend towards the infinite-order PCFB as the number of iterations in increased. The algorithm is based on a polynomial cross-spectral density (CSD) matrix which for the subband coder design possesses a specific structure, incorporating both parahermitian and pseudo-circulant properities. We therefore demonstrate how these implicit properties can be exploited to enhance the estimation accuracy of the CSD matrix.

In Sec. II, we present a review of relevant theory and results pertaining to filter bank design and the extension of the EVD to polynomial matrices. A review of the SBR2 algorithm is given as a technique for computing the polynomial EVD. In Sec. III, a new cost function for SBR2 is introduced, which improves the energy compaction ability of the algorithm. The applicability of the modified SBR2 to the problem of data compression is investigated in Sec. IV. This leads to a method by which a priori knowledge about the subband signals is used to improve the decorrelation performance of this algorithm. The resulting technique is compared to the state-of-the-art, IGA [20], the AEVD [24] and the computationally efficient window method [17] in Sec. V. We present experimental results which suggest that our algorithm outperforms the window method, IGA and AEVD on a set of benchmark problems for

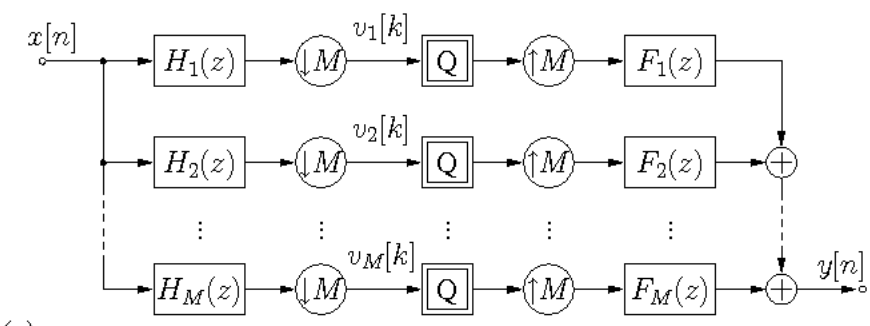

(a)

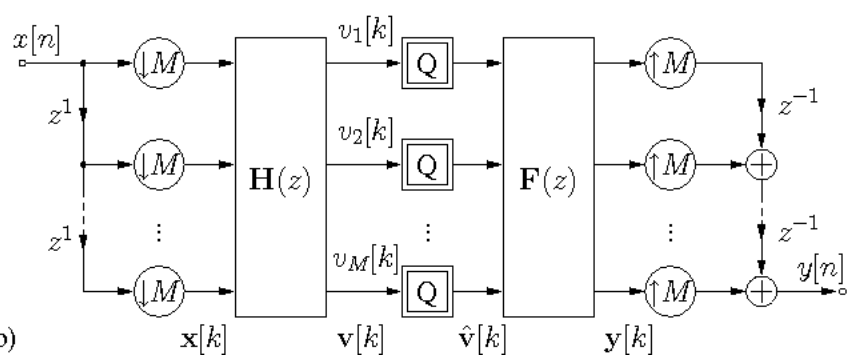

Fig. 1. (a) $M$-channel uniform, maximally decimated filter bank and (b) its equivalent polyphase represention.

moderate to large filter orders. Finally, conclusions are given in Sec. VI.

\section{Optimal Filter Banks ANd Polynomial Matrix DECOMPOSITIONS}

\section{A. Preliminary}

A polynomial matrix is a matrix whose elements are polynomials, or equivalently a polynomial with matrix coefficients [29]. In this paper, we will use the term polynomial to include Laurent polynomials which can include negative powers of the indeterminate variable. We denote a $P \times Q$ polynomial matrix in the indeterminate variable $z^{-1}$ by

$$
\mathbf{A}(z)=\sum_{\tau=\tau_{1}}^{\tau_{2}} \mathbf{A}[\tau] z^{-\tau}=\left[\begin{array}{ccc}
a_{11}(z) & \ldots & a_{1, Q}(z) \\
\vdots & \ddots & \vdots \\
a_{P, 1}(z) & \ldots & a_{P, Q}(z)
\end{array}\right]
$$

where $\tau \in \mathbb{Z}, \tau_{1} \leq \tau_{2}$, and

$$
a_{p q}(z)=\sum_{\tau=\tau_{1}}^{\tau_{2}} a_{p q}[\tau] z^{-\tau}
$$

with $a_{p q}[\tau] \in \mathbb{C} \forall p, q, \tau$. Since the leading term of $z^{\tau_{1}} \mathbf{A}(z)$ is constant, the effective order of $\mathbf{A}(z)$ is $\tau_{2}-\tau_{1}$. A transform pair as in (1) is denoted as $\mathbf{A}(z) \bullet-\circ \mathbf{A}[\tau]$.

\section{B. Filter Bank Optimality}

A typical model of a subband coder is the $M$-channel, maximally decimated, uniform filter bank shown in Fig. 1(a) and its polyphase form [12] shown in Fig. 1(b). It consists of an analysis bank followed by subband processors $\left\{q_{k}\right\}$, applied to the subband signals, and a synthesis bank. The subband processors $\left\{q_{k}\right\}$ are typically scalar quantisers. If $\mathbf{F}(z)$ in Fig. 1(b) is chosen such that $\mathbf{F}(z) \mathbf{H}(z)=c z^{-\tau} \mathbf{I}$, for some constant $c$ and integer $\tau$, then the subband coder is a perfect reconstruction filter bank, which in the absence of any subband processing is transparent from input to output, i.e. 
$y[n]=c x[n-\tau]$. Furthermore, an elegant and simple reconstruction may be obtained, if the matrix $\left.\mathbf{H}(z)\right|_{z=e^{j \Omega}}=\mathbf{H}\left(e^{j \Omega}\right)$ is unitary for all normalised angular frequencies $\Omega$; that is, $\mathbf{H}(z)$ satisfies the PU or orthonormality condition [12]

$$
\mathbf{H}(z) \tilde{\mathbf{H}}(z)=\tilde{\mathbf{H}}(z) \mathbf{H}(z)=\mathbf{I},
$$

where $\mathbf{H}(z)$ is an $M \times M$ polynomial matrix and $\tilde{\mathbf{H}}(z)$ is the paraconjugate transpose of $\mathbf{H}(z)$, i.e. $\tilde{\mathbf{H}}(z)=\mathbf{H}^{\mathrm{H}}\left(z^{-1}\right)$. In this case, the synthesis bank is simply given by $\mathbf{F}(z)=$ $\tilde{\mathbf{H}}(z)$. Another important property of paraunitary filter banks is their losslessness, which means that the total signal power at every frequency is conserved by the transformation [12], i.e. $\mathbf{H}(z)$ defines an all-pass filter. Our discussions are limited to maximally decimated, uniform paraunitary filter banks.

The design of an optimal PU subband coder, for a given fixed budget of quantiser bits, consists of simultaneously optimising the analysis and synthesis filters as well as choosing a subband bit allocation strategy such that the reconstruction error is minimised. The scalar input signal $x[n]$ is typically assumed to be a zero mean, wide-sense stationary (WSS) random process with a known power spectrum $S_{x x}\left(e^{j \Omega}\right)$. This is equivalent to saying that the $M$-fold blocked input signal vector $\mathbf{x}[k]$ is jointly WSS [12], where $k=M n$ denotes the low-rate time index. Assuming a high bit rate for the quantiser and optimum bit allocation, an optimal PU filter bank maximises the well-known coding gain [8]

$$
G=\sigma_{x}^{2}\left(\prod_{m=1}^{M} \sigma_{m}^{2}\right)^{-\frac{1}{M}},
$$

where $\sigma_{x}^{2}$ is the variance of $x[n]$ and $\sigma_{m}^{2}$ is the variance of the $m$ th subband signal $v_{m}[k]$. The denominator is the geometric mean of the subband variances. Since $\sigma_{x}^{2}$ is fixed, the optimisation of the analysis filters consists of minimising this geometric mean under the orthonormality condition. For the unconstrained filter order case, Vaidyanathan derives a set of necessary and sufficient conditions for optimality of a PU filter bank [8]:

1) strong (or total) decorrelation - the subband signals are decorrelated at all relative time lags, i.e. $\mathcal{E}\left\{v_{m}[k] v_{p}^{*}[k-\tau]\right\}=\delta(m-p) \delta(\tau) \forall m, p, \tau$, where $\mathcal{E}\{\cdot\}$ denotes the expectation operator, $a^{*}$ the complex conjugation of $a$, and $\delta(\cdot)$ the Kronecker delta function;

2) spectral majorisation - the set of subband spectra $S_{m m}\left(e^{j \Omega}\right)$ has the property $S_{11}\left(e^{j \Omega}\right) \geq S_{22}\left(e^{j \Omega}\right) \geq$ $\ldots \geq S_{M M}\left(e^{j \Omega}\right) \forall \Omega$, where the subbands are numbered such that $\sigma_{m}^{2} \geq \sigma_{m+1}^{2}$. In other words, the PSD matrix $\mathbf{S}_{x x}\left(e^{j \Omega}\right)$ of $\mathbf{x}[k]$ is diagonalised for every $\Omega$ such that the eigenvalues of $\mathbf{S}_{x x}\left(e^{j \Omega}\right)$ are arranged in decreasing order [1].

A procedure for obtaining optimal PU systems, for the unconstrained order case, is presented in [8]. We can also obtain an optimal PU system through the design of a PCFB, which is defined as follows: Consider that $M-K$ channels are discarded in the synthesis part of the $M$-channel filter bank in Fig. 1. A filter bank that minimises the average mean square reconstruction error for all $K$ is called a PCFB. The set of subband variances $\sigma_{m}^{2}$ generated by a PCFB is said to majorise any other possible set of subband variances. It turns out that the orthonormal PCFB is also optimal for energy compaction: the subband variances are arranged as $\sigma_{1}^{2} \geq \sigma_{2}^{2} \geq \ldots \geq \sigma_{K}^{2}$, such that $\sum_{m=1}^{K} \sigma_{m}^{2}, 1 \leq K<M$ is maximised. For the case $K=1, \sigma_{1}^{2}$ is maximised by $H_{1}(z)$, which is an optimal compaction filter. Furthermore, since the filters of a PCFB are orthonormal, $H_{1}(z)$ satisfies the Nyquist- $M$ constraint, viz.,

$$
\frac{1}{M} \sum_{i=0}^{M-1}\left|H_{1}\left(e^{j\left(\Omega-\frac{2 \pi i}{M}\right)}\right)\right|^{2}=1
$$

where $H_{1}\left(e^{j \Omega}\right)$ is a real coefficient FIR filter [12]. A quantitative measure of energy compaction performance is given by the compaction gain [17],

$$
G_{\mathrm{comp}}=\frac{\sigma_{1}^{2}}{\sigma_{x}^{2}}
$$

which is maximised by a compaction filter, i.e. the first filter of a PCFB.

\section{Polynomial Matrix EVD}

Given a vector of signals $\mathbf{x}[k] \in \mathbb{C}^{M}$, compression can be achieved by exploiting redundancy in the form of correlation between the $M$ signals $x_{m}[k], m=1 \ldots M$ constituting $\mathbf{x}[k]$. If these signals are only correlated at zero relative timelag, then the Karhunen-Loeve transform (KLT) matrix $\mathbf{H}$ can perform decorrelation

$$
\mathbf{v}[k]=\mathbf{H} \mathbf{x}[k],
$$

whereby $\mathbf{H}$ is derived from an EVD of the covariance matrix $\mathbf{R}=\mathcal{E}\left\{\mathbf{x}[k] \mathbf{x}^{\mathrm{H}}[k]\right\}=\mathbf{H}^{\mathrm{H}} \mathbf{C H}$, with $\mathbf{C}$ diagonal and $\mathbf{H}$ unitary matrices, or from an SVD operating on the data matrix. The decorrelation according to (7) converts the form of the redundancy from correlation between the signals to disparity between the signal powers. Compression is realised by discarding low power channels which lie in the noise-only subspace estimated by the KLT.

If signals in $\mathbf{x}[k]$ are correlated for lags other than lag zero, then the KLT can only achieve instantaneous decorrelation, and not strong decorrelation as defined in Sec. II-B. While this problem occurs in many techniques such as separation of convolutively mixed signals, we here concentrated on the subband coding idea, where $\mathbf{x}[k]$ is obtained by demultiplexing an input signal $x[n]$ as shown in Fig. 1. We would like to find a polyphase matrix $\mathbf{H}[k]$ with z-transform $\mathbf{H}(z) \bullet-\circ \mathbf{H}[k]$, such that the transformed data vector $\mathbf{v}[k]$

$$
\mathbf{v}[k]=\sum_{k=-\infty}^{\infty} \mathbf{H}[\kappa] \mathbf{x}[k-\kappa]
$$

is strongly decorrelated. This requires the cross-spectral density (CSD) matrix $\mathbf{C}(z)$ of the transformed signals $\mathbf{v}[k] \in \mathbb{C}^{M}$ to be diagonalised, such that the polynomial matrix $\mathbf{C}(z)$ is given by

$$
\mathbf{C}(z)=\mathbf{H}(z) \mathbf{R}(z) \tilde{\mathbf{H}}(z)=\operatorname{diag}\left\{c_{11}(z) \ldots c_{M M}(z)\right\}
$$


where $\mathbf{R}(z) \bullet-\circ \mathbf{R}[\tau]$ is the CSD matrix based on the covariance matrix $\mathbf{R}[\tau]=\mathcal{E}\left\{\mathbf{x}[k] \mathbf{x}^{\mathrm{H}}[k-\tau]\right\}$ of the input data vector, and $\mathbf{C}(z)$ is defined likewise based on $\mathbf{v}[k]$.

We refer to (9) as a polynomial EVD (PEVD) and demand as a generalisation of the EVD that the polynomial matrix $\mathbf{H}(z)$ is constrained to satisfy the PU condition in (3).

\section{PCFBs and PEVDs}

To explore the energy compaction property of the PEVD w.r.t. data compression, we assume that only the first $P \leq M$ signals of the $M$-element transformed data vector $\mathbf{v}[k]$ in (8) are retained,

$$
\hat{\mathbf{v}}[k]=\operatorname{diag}\{\underbrace{1 \cdots 1}_{P} \underbrace{0 \cdots 0}_{M-P}\} \mathbf{v}[k] .
$$

The total power of this compressed signal vector $\hat{\mathbf{v}}[k]$,

$$
\sigma_{\hat{v}}^{2}=\sum_{p=1}^{P} c_{p p}[0],
$$

is maximised for each $P \leq M$. The terms $c_{p p}[\tau], p=1 \ldots M$, are the diagonal entries of the covariance matrix $\mathbf{C}[\tau]$ corresponding to the CSD $\mathbf{C}(z)$ in (9). By applying the PU matrix $\tilde{\mathbf{H}}(z)$ to $\hat{\mathbf{v}}[k]$, as shown in Fig. 1(b) with $\mathbf{F}(z)=\tilde{\mathbf{H}}(z)$,

$$
\mathbf{y}[k]=\sum_{k=-\infty}^{\infty} \mathbf{H}^{\mathrm{H}}[-\kappa] \hat{\mathbf{v}}[k-\kappa],
$$

represents an approximate reconstruction of $\mathbf{x}[k]$, whereby $\tilde{\mathbf{H}}(z) \bullet-\circ \mathbf{H}^{\mathrm{H}}[-k]$.

Since (11) is maximised, the sum of the variances of the $M-P$ signals suppressed in (10) is minimised. With $\mathbf{H}(z)$ being PU, the power $\sigma_{e}^{2}=\sum_{m=P+1}^{M} c_{m m}[0]$ of the reconstruction error $\mathbf{y}[k]-\mathbf{x}[k]$ is also minimised. Therefore, the PU matrix $\mathbf{H}(z)$ responsible for this minimisation can be interpreted as an extension of the PCFB for subband coding, motivating the need for a PEVD.

\section{E. Polynomial EVD via the Sequential Best Rotation Algo- rithm (SBR2)}

A number of algorithms have been reported to approximate the PEVD factorisation of (9) in [21], [22], [24], [27]. We review the sequential best rotation (SBR2) approach [22], which will form the basis of the proposed subband coding scheme in Sec. III.

An iterative approach to obtain the decomposition (9) is described in [22]. This method calculates a sequences of paraunitary operations consisting of delays and rotations to iteratively eliminate the largest off-diagonal terms in the CSD matrix. The approach in [21], [22] is based on second order statistics, and therefore termed second order sequential best rotation (SBR2) algorithm.

After $L$ iterations, SBR2 is set to achieve the decomposition

$$
\begin{aligned}
\mathbf{S}_{L}(z) & =\mathbf{H}_{L}(z) \hat{\mathbf{R}}(z) \tilde{\mathbf{H}}_{L}(z) \\
& \approx \operatorname{diag}\left\{s_{L, 11}(z) s_{L, 22}(z) \ldots s_{L, M M}(z)\right\}
\end{aligned}
$$

where $\hat{\mathbf{R}}(z)$ is an estimate of the CSD matrix $\mathbf{R}(z)$ based on the available samples of $\mathbf{x}(z)$, and $\mathbf{S}_{L}(z)$ provides an estimate of the approximately diagonalised CSD matrix $\mathbf{C}(z)$ of the transformed signals $\mathbf{v}(z)$.

SBR2 starts by setting $\mathbf{S}_{0}(z)=\hat{\mathbf{R}}(z)$. In each subsequent step $l$, SBR2 will eliminate the largest off-diagonal element of $\mathbf{S}_{l-1}(z)$. This element can be identified by its coordinates $m_{l}, p_{l}$ and lag $\tau_{l}$,

$$
\left\{m_{l}, p_{l}, \tau_{l}\right\}=\underset{m, p, m \neq p, \tau}{\arg \max }\left|s_{l-1, m p}[\tau]\right| \quad,
$$

where $s_{l-1, m p}[\tau]$ is the element in row $m$ and column $p$ of the covariance matrix $\mathbf{S}_{l-1}[\tau]$ corresponding to $\mathbf{S}_{l-1}(z)$. The elimination of this element is performed by the generalised similarity transform

$$
\mathbf{S}_{l}(z)=\mathbf{P}_{l}(z) \mathbf{S}_{l-1}(z) \tilde{\mathbf{P}}_{l}(z),
$$

whereby the elementary PU matrix

$$
\mathbf{P}_{l}(z)=\mathbf{Q}_{l} \boldsymbol{\Lambda}_{l}(z)
$$

consists of a delay matrix $\boldsymbol{\Lambda}(z)$ and a Jacobi rotation $\mathbf{Q}$. The delay matrix shifts the largest off-diagonal element of $\mathbf{S}_{l=1}[\tau]$ onto lag zero by setting

$$
\boldsymbol{\Lambda}_{l}(z)=\operatorname{diag}\{\underbrace{1 \cdots 1}_{p_{l}-1} z^{-\tau_{l}} \underbrace{1 \cdots 1}_{M-p_{l}}\} .
$$

The operation $\boldsymbol{\Lambda}_{l}(z) \mathbf{S}_{l-1}(z) \tilde{\boldsymbol{\Lambda}}_{l}(z)$ will transfer the largest offdiagonal element onto the lag-zero slice. The Jacobi rotation $\mathbf{Q}_{l}$,

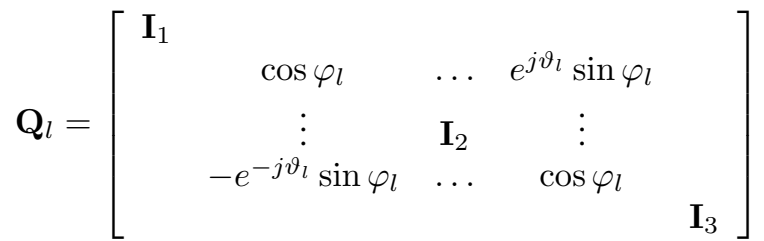

with $\mathbf{I}_{i}$ identity matrices of dimensions $\left(\min \left\{m_{l}, p_{l}\right\}-1\right)$, $\left(\left|m_{l}-p_{l}\right|-1\right)$ and $\left(M-\max \left\{m_{l}, p_{l}\right\}+1\right)$, respectively, for $i=1,2,3$,contains angles $\varphi_{l}$ and $\vartheta_{l}$ selected to eliminate the largest off-diagonal element and transfer its energy onto the main diagonal [10]. The transfer is performed such that the larger element lies higher up on the diagonal, which during the iteration process leads to spectral majorisation.

The iteration continues until $\mathbf{S}_{L}(z)$ is sufficiently diagonalised, such that the maximum off-diagonal element

$$
\max _{m, p, m \neq p, \tau}\left|s_{L, m p}[\tau]\right| \leq \epsilon
$$

falls below a pre-selected threshold $\epsilon$. In this case, $\mathbf{S}_{L}(z)$ is the approximately diagonalised CSD matrix of (13), and the paraunitary matrix $\mathbf{H}_{L}(z)$ in (13) is given by

$$
\mathbf{H}_{L}(z)=\mathbf{P}_{L}(z) \mathbf{P}_{L-1}(z) \ldots \mathbf{P}_{1}(z) \quad .
$$

The accuracy of this decomposition depends on $\epsilon$, the sample size over which $\hat{\mathbf{R}}(z)$ is estimated as an approximation of the true CSD matrix $\mathbf{R}(z)$, as well as optimality of the estimation procedure to be discussed in Sec. IV.

The order of both $\mathbf{H}_{L}(z)$ and $\mathbf{S}_{L}(z)$ can grow significantly with the number of iterations $L$. Therefore, a trim function was proposed in [22] to truncate the highest order coefficient matrices at each SBR2 stage based on a small threshold, and thus limit the increase in order on both polynomial matrices. 


\section{Modified SBR2 (SBR2C) AlgORITHM}

There is an important limitation of the search strategy employed by the SBR2 algorithm described in Sec. II. The parameter values for each delay-rotation matrix are chosen using a generalisation of the classical Jacobi algorithm [10]. This method may be viewed as a "greedy" optimisation scheme that annihilates the largest cross-correlation at each algorithm iteration. Therefore, SBR2 is proportionately more sensitive to changes (in correlation) in dominant signals than it is to changes in weak signals. This is because, in general, the largest cross-correlation coefficients occur between dominant signals, which are minimised by the algorithm at every step. The detrimental effect on algorithm performance when operating on estimated CSD matrices is twofold:

1) The extent to which polynomial matrix diagonalisation (strong decorrelation) is carried out is restricted: after a number of iterations, the algorithm begins to zero noise-related cross-correlations between dominant signals rather than true signal-related cross-correlations between weaker signals.

2) The extent to which spectral majorisation is performed is limited: Energy in weaker, correlated signals is not compacted into as few channels as possible. This is usually because energies due to cross-correlation terms, which are spread among pairs of weak signals, are not transferred to the auto-correlation of the signals.

The above problems can be alleviated by the use of a cost function which is proportionately equally sensitive to changes in any of the signals. The coding gain in (4) for subband coding has this property. Here, we define a new cost function simply by substituting the variance terms in the coding gain definition (4) by variances $s_{l, m m}[0]$ obtained after $l$ diagonalisation steps, using e.g. SBR2, and based on sample statistics in $\mathbf{S}_{0}(z)=\hat{\mathbf{R}}(z)$, giving

$$
\hat{G}_{l}=\left(\frac{1}{m} \sum_{m=1}^{M} s_{l, m m}[0]\right) \cdot\left(\prod_{m=1}^{M} s_{l, m m}[0]\right)^{-\frac{1}{M}} .
$$

As with the coding gain, minimisation of the product of the transformed signal variances in (22) leads to maximal energy compaction and spectral majorisation. In this paper, the SBR2 algorithm with this cost function is referred to as the SBR2 algorithm modified for subband coding (SBR2C). We now make the following assertion.

Theorem 1: The cost function $\hat{G}_{l}$ in (22) is maximised at each step $l$ of the SBR2C algorithm if the largest normalised magnitude squared off-diagonal term with indices $m_{l}, p_{l}$, and $\tau_{l}$,

$$
\left\{m_{l}, p_{l}, \tau_{l}\right\}=\underset{m, p, m \neq p, \tau}{\arg \max } \frac{\left|s_{l-1, m p}[\tau]\right|^{2}}{s_{l-1, m m}[0] s_{l-1, p p}[0]}
$$

in $\mathbf{S}_{l-1}[\tau]$ is zeroed.

Proof: The indices $m_{l}$ and $p_{l}$ identify the two signals involved in the elementary similarity transformation $\mathbf{P}_{l}(z)$ in (16) at step $l$. Note that the numerator in (22) - i.e. the arithmetic mean of sample variances - can be treated as a constant $\kappa_{1}$ under PU operations and therefore throughout the SBR2 algorithm. Further, since signals indexed $m_{l}$ and $p_{l}$ are the only ones modified by the transformation in question, we may write

$$
\hat{G}_{l}=\kappa_{1}\left(\kappa_{2, l} s_{l, m_{l} m_{l}}[0] s_{l, p_{l} p_{l}}[0]\right)^{-\frac{1}{M}},
$$

where $\kappa_{2, l}$ represents the product of the $P-2$ sample variances that are invariant under the $l$ th elementary PU transformation. Following the transformation process, we have

$$
\left(\frac{\hat{G}_{l}}{\hat{G}_{l-1}}\right)^{M}=\frac{s_{l-1, m_{l} m_{l}}[0] s_{l-1, p_{l} p_{l}}[0]}{s_{l, m_{l} m_{l}}[0] s_{l, p_{l} p_{l}}[0]},
$$

but from properties of the transformation it can easily be shown that

$s_{l, m_{l} m_{l}}[0] s_{l, p_{l} p_{l}}[0]=s_{l-1, m_{l} m_{l}}[0] s_{l-1, p_{l} p_{l}}[0]-\left|s_{l-1, m_{l} p_{l}}\left[\tau_{l}\right]\right|^{2}$,

and therefore

$$
\left(\frac{\hat{G}_{l}}{\hat{G}_{l-1}}\right)^{M}=\frac{s_{l-1, m_{l} m_{l}}[0] s_{l-1, p_{l} p_{l}}[0]}{s_{l-1, m_{l} m_{l}}[0] s_{l-1, p_{l} p_{l}}[0]-\left|s_{l-1, m_{l} p_{l}}\left[\tau_{l}\right]\right|^{2}} .
$$

It follows that for any one iteration of the algorithm, the cost function $\hat{G}_{l}$ is maximised by choosing the correct set $\left\{m_{l}, p_{l}, \tau_{l}\right\}$ at the $l$ th iteration such that the objective function

$$
J=\frac{\left|s_{l-1, m_{l} p_{l}}\left[\tau_{l}\right]\right|^{2}}{s_{l-1, m_{l} m_{l}}[0] s_{l-1, p_{l} p_{l}}[0]}
$$

is as close to unity as possible. The denominator of (28) can be viewed as a normalisation factor that essentially stabilises the algorithm. In essence, this is because large cross-covariance coefficients due to strong signals, say, are attenuated relative to those associated with weaker signals. Hence, the maximisation of $\hat{G}_{l}$ entails a generalised classical Jacobi search for the largest normalised cross-covariance term.

There are two modifications that need to be made to the SBR2 algorithm in order to obtain the modified SBR2 algorithm. The first is that the correlation based objective function $\left|s_{l-1, m p}[\tau]\right|^{2}$ in (15) is replaced by the quotient

$$
J_{l-1}=\frac{\left|s_{l-1, m p}[\tau]\right|^{2}}{s_{l-1, m m}[0] s_{l-1, p p}[0]}
$$

based on (28), searching over all normalised cross-correlation functions. Secondly, the stopping criterion $\epsilon$ in (20) needs to be defined in terms of $J_{L}$ in (29) rather than $\left|s_{L, m p}\left[\tau_{L}\right]\right|^{2}$.

The proposed modified cost function in (29) improves the strong decorrelation and spectral majorisation performances of the SBR2 algorithm. Hence, the SBR2C algorithm is more suited to the applications of data compression and broadband subspace decomposition than its correlation-based counterpart. Note that the algorithm intrinsically aims to design a filter bank that is optimal for multichannel data compression because its optimisation is exclusively geared towards the maximisation of energy compaction. The proof of convergence for SBR2 in [22] no longer holds for the modified SBR2 algorithm, since the condition $J_{l} \rightarrow \infty$ will arise if either $s_{l, m m}[0]$ or $s_{l, p p}[0]$ tend to zero. However, the original proof remains valid if individual signal powers are artificially bounded from below by a small noise-related offset value. 


\section{Cross-Spectral Density Matrix Estimation}

The SBR2 algorithm and its proposed modificiation, the SBR2C algorithm, can be classed as "blind" techniques since they require knowledge of neither the source signals nor their mixing. However, these algorithms require the CSD matrix $\mathbf{R}(z)$ of the demultiplexed data vector $\mathbf{x}[k]$. In practise, an estimate of this CSD matrix $\hat{\mathbf{R}}(z)$ has to be calculated based on a finite window of data. The accuracy of this estimate is therefore crucial for the performance of subband coding. Below, we first review two important properties of the CSD matrix in Sec. IV-A, in order to suggest two procedures in Sec. IV-B that implicitly exploit the CSD matrix' properties and therefore yield enhanced estimates.

\section{A. Cross-Spectral Density Matrix}

Consider the subband coder in Fig. 1(b). We assume that the scalar input signal $x[n]$ is a zero mean, WSS random process [9]. The blocked filter bank input vector is given by

$$
\mathbf{x}[k]=\left[x_{1}[k] x_{2}[k] \cdots x_{M}[k]\right]^{\mathrm{T}}
$$

where $x_{m}[k]=x[M n+m-1], 1 \leq m \leq M$ are the demultiplexed signals. The $M \times M$ CSD matrix $\mathbf{R}(z)$,

$$
\mathbf{R}(z)=\sum_{\tau=-\infty}^{\infty} \mathbf{R}[\tau] z^{-1}=\sum_{\tau=-\infty}^{\infty} \mathcal{E}\left\{\mathbf{x}[k] \mathbf{x}^{\mathrm{H}}[k-\tau]\right\} z^{-1},
$$

has the form

$$
\mathbf{R}(z)=\left[\begin{array}{cccc}
r_{1,1}(z) & r_{1,2}(z) & \ldots & r_{1, M}(z) \\
r_{2,1}(z) & r_{2,2}(z) & \ldots & r_{2, M}(z) \\
\vdots & & \ddots & \vdots \\
r_{M, 1}(z) & \ldots & r_{M, M-1} & r_{M, M}(z)
\end{array}\right],
$$

where

$$
r_{m, p}[\tau]=\mathcal{E}\left\{x_{m}[k] x_{p}^{*}[k-\tau]\right\}
$$

is the cross-correlation sequence between subband signals $x_{m}[k]$ and $x_{p}[k]$. The CSD matrix $\mathbf{R}(z)$ has the following important properties:

1) Para-Hermitian Property. From the definition of the cross correlation function $r_{m, p}[\tau]=$ $\mathcal{E}\left\{x_{m}[k] x_{p}^{*}[k-\tau]\right\}=\mathcal{E}\left\{x_{p}[k+\tau] x_{m}^{*}[k]\right\}^{*}=$ $r_{p, m}^{*}[-\tau]$ and $r_{m, p}(z)=\sum_{\tau=-\infty}^{\infty} r_{m, p}[\tau] z^{-\tau}=$ $\tilde{r}_{p, m}(z)$, it follows that $\tilde{\mathbf{R}}(z)=\mathbf{R}(z)$.

2) Pseudo-circulant Property. An $M \times M$ polynomial matrix $\mathbf{R}(z)$ with entries $r_{m, p}(z)$ as defined in (32) is pseudo-circulant if there exist polynomials $\phi_{0}(z), \phi_{1}(z)$, $\ldots \phi_{M-1}(z)$ such that [12]

$$
r_{m, p}(z)= \begin{cases}\phi_{p-m}(z), & 1 \leq m \leq p \leq M \\ z^{-1} \phi_{p-m+M}(z), & 1 \leq p<m \leq M\end{cases}
$$

The pseudo-circulant property of $\mathbf{R}(z)$ means that each row is derived from the previous one by right-shifting elements and forming a wrap-around with a multiplication by $z^{-1}$,

$$
\mathbf{R}(z)=\left[\begin{array}{cccc}
\phi_{0}(z) & \phi_{1}(z) & \ldots & \phi_{M-1}(z) \\
z^{-1} \phi_{M-1}(z) & \phi_{0}(z) & \ldots & \phi_{M-2}(z) \\
\vdots & \ddots & \ddots & \vdots \\
z^{-1} \phi_{1}(z) & \ldots & z^{-1} \phi_{M-1}(z) & \phi_{0}(z)
\end{array}\right]
$$

whereby here specifically $\phi_{m}[\tau], m=0 \cdots(M-1)$, are the $M$ polyphase components of the autocorrelation function of $x[n]$. The pseudo-circulant property of $\mathbf{R}(z)$ is intimated but not explicitly derived in [12]; since it is central to the estimation of $\hat{\mathbf{R}}(z)$ below, (35) is shown in Appendix A.

\section{B. Estimation of $\mathbf{R}(z)$}

Based on the availability of a finite window $T$ of input data $x[n], 0 \leq n \leq T$, we discuss a direct approach that yields a suboptimal estimation of $\hat{\mathbf{R}}(z)$, followed by two estimates that exploit the properties of $\mathbf{R}(z)$ and lead to enhanced accuracy.

1) Subband-Based Calculation of $\hat{\mathbf{R}}(z)$ : If $\mathbf{x}[k]$ is ergodic, (31) can be converted to estimate $\mathbf{R}(z)$ via

$$
\hat{\mathbf{R}}^{(1)}(z)=\sum_{\tau=-T / M}^{T / M} \frac{M}{T} \sum_{k=0}^{T / M} \mathbf{x}[k] \mathbf{x}^{\mathrm{H}}[k-\tau] z^{-\tau} .
$$

In this case, every entry of $\hat{\mathbf{R}}^{(1)}(z)$ is estimated from $T / M$ samples. If the influence of marginal values of $x[n]$ is neglected, $\mathbf{R}(z)$ will be para-Hermitian. However, every entry along the diagonals of $\hat{\mathbf{R}}^{(1)}(z)$ is estimated from a different data set, and the pseudo-circulant property according to (35) will only be achieved in the limit $T \rightarrow \infty$.

2) Improved Subband-Based Calculation: An improved estimate $\hat{\mathbf{R}}^{(2)}(z)$ can be obtained by forcing it to be pseudocirculant. If $\hat{\phi}_{m}^{(2)}[\tau]$ is a cross-correlation entry of $\hat{\mathbf{R}}^{(2)}(z)$, reflecting the structure in (35), it can be obtained by averaging across the diagonals of $\hat{\mathbf{R}}^{(1)}(z)$ in (36). With the entries $\hat{r}_{m, p}^{(1)}[\tau]$ of $\hat{\mathbf{R}}^{(1)}(z)$,

$\hat{\phi}_{m}^{(2)}[\tau]=\frac{1}{M}\left(\sum_{p=1}^{M-m} \hat{r}_{p, p+m}^{(1)}[\tau]+\sum_{p=M-m+1}^{M} \hat{r}_{p, p+m-M}^{(1)}[\tau+1]\right)$,

for $m=0 \cdots(M-1)$. Note that compared to $\hat{\mathbf{R}}^{(1)}(z)$, every entry of $\hat{\mathbf{R}}^{(2)}(z)$ is now an estimate drawn from $T$ rather than $T / M$ samples.

3) Calculation Based on Input Auto-Correlation: A third option is to draw the elements of the estimate $\hat{\mathbf{R}}(z)$ directly from an estimate of the auto-correlation sequence of $x[n]$,

$$
\hat{\phi}^{(3)}(z)=\sum_{\tau=-T}^{T} \frac{1}{T} \sum_{n=0}^{T} x[n] x^{*}[n-\tau] z^{-\tau},
$$

to form an estimate $\hat{\mathbf{R}}^{(3)}(z)$ according to (35) by splitting $\hat{\phi}^{(3)}(z)$ in (38) into its $M$ polyphase components $\hat{\phi}_{m}^{(3)}(z), m=$ $0 \cdots(M-1)$.

Alternatively, $\hat{\mathbf{R}}^{(3)}(z)$ can be constructed in closed form from (38), in analogy to FIR block filtering [34], as

$$
\hat{\mathbf{R}}^{(3)}\left(z^{M}\right)=\mathbf{D}(z) \mathbf{T} \hat{\boldsymbol{\Phi}}^{(3)}(z) \mathbf{T}^{\mathrm{H}} \tilde{\mathbf{D}}(z),
$$


whereby

$$
\begin{aligned}
\hat{\mathbf{\Phi}}^{(3)}(z) & =\left[\begin{array}{c}
\hat{\phi}^{(3)}(z) \\
\hat{\phi}^{(3)}\left(z e^{j \frac{2 \pi}{M}}\right) \\
\ddots \\
\hat{\phi}^{(3)}\left(z e^{j \frac{2 \pi}{M}(M-1)}\right)
\end{array}\right] \\
\mathbf{D} & =\operatorname{diag}\left\{1 z^{-1} \ldots z^{-M+1}\right\}
\end{aligned}
$$

and $\mathbf{T}$ is a unitary $M \times M$ DFT matrix normalised by $\sqrt{\frac{1}{M}}$.

The CSD estimate $\hat{\mathbf{R}}^{(3)}(z)$ is based on $T$ samples for every entry, and provides the same accuracy and result as $\hat{\mathbf{R}}^{(2)}(z)$. When using any subband coder in the following results section, it is always assumed that it operates on an estimate provided by the latter two optimal methods discussed in this section.

\section{Simulations and Results}

To demonstrate the performance of the proposed SBR2C algorithm, we define two simulation scenarios in Sec. V-A. Simulations on the achievable coding gain are presented in Sec. V-B, followed by comments on the influence of estimation inaccuracies in Sec. $\mathrm{V}-\mathrm{C}$ and the algorithm and encoder complexity in Sec. V-D.

\section{A. Simulation Scenarios}

In general, we assume subband coding of a signal $x[n]$ which can be modelled as the output of an innovation filter [31] excited by uncorrelated noise. Popular processes for subband coding use autoregressive (AR) models of first and second order - AR(1) [13], [16], [17] and AR(2) [13], [16], [18] — or higher order models such as $\operatorname{AR}(4)$ [19], [20], AR(5) [13], [18], and ARMA(5) [32] providing a multiband structure. For this reason, we utilise an $\operatorname{AR}(4)$ process characterised by a transfer function $A(z)$ with two complex conjugate pole pairs $0.9 e^{ \pm j 0.6283}$ and $0.85 e^{ \pm j 2.8274}$, and magnitude response shown in Fig. 2(a).

Further, an ensemble of 330 moving average (MA) systems of order 14 is utilised, whereby the coefficients of sample systems are drawn from independent and identically distributed Gaussian processes of unit variance and zero mean. The excitation of the innovation filters are formed by uncorrelated zero mean and unit variance quaternary phase shift keying (QPSK) sequences. With the exception of the window method, all algorithms below operate on $x[n]$ decomposed into $M=4$ polyphase components.

\section{B. Coding Gain}

1) PCFB Approximation and Spectral Majorisation : Multiplexing the AR(4) process $x[n]$ into $M=4$ polyphase components for subband coding, Figs. 2 and 3 show the results for the proposed SBR2 system as well as for SBR2, IGA, and AEVD in terms of the filter bank filters and the spectral majorisation. The CSD matrix estimate $\hat{\mathbf{R}}(z)$ for SBR2C, SBR2, and AEVD, and the estimate of the power spectral density $S_{x x}\left(e^{j \Omega}\right)$ required for IGA are based on $2^{11}$ samples of $x[n]$.

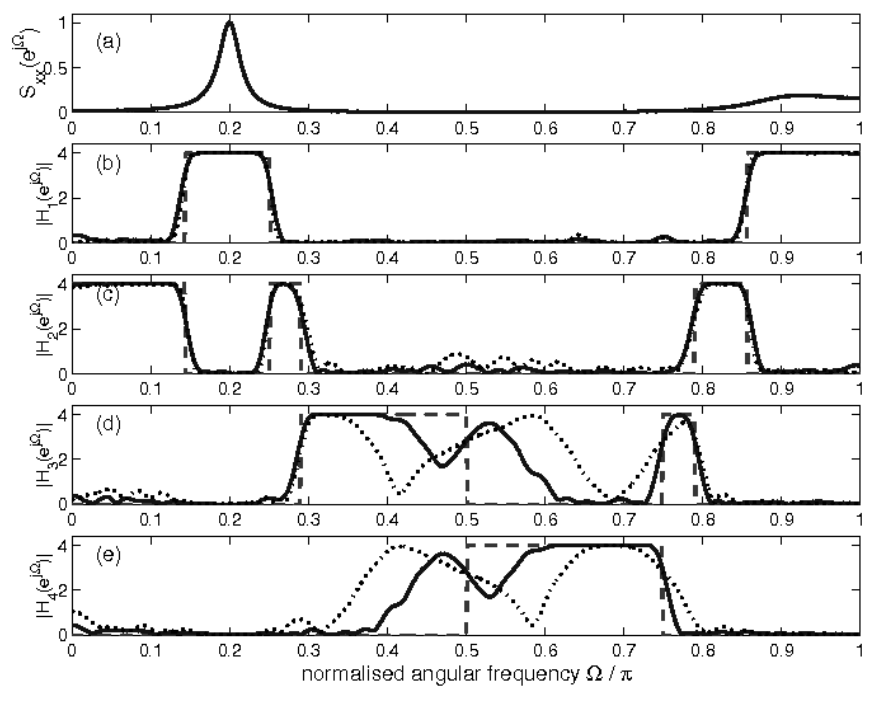

Fig. 2. (a) PSD of input signal $x[n]$ produced by AR(4) model, and (b)-(e) magnitude responses of filters $H_{m}(z)$ for theoretical PCFB (dashed), SBR2C (solid, $L=150$ iterations) and SBR2 (dotted, $L=150$ iterations).

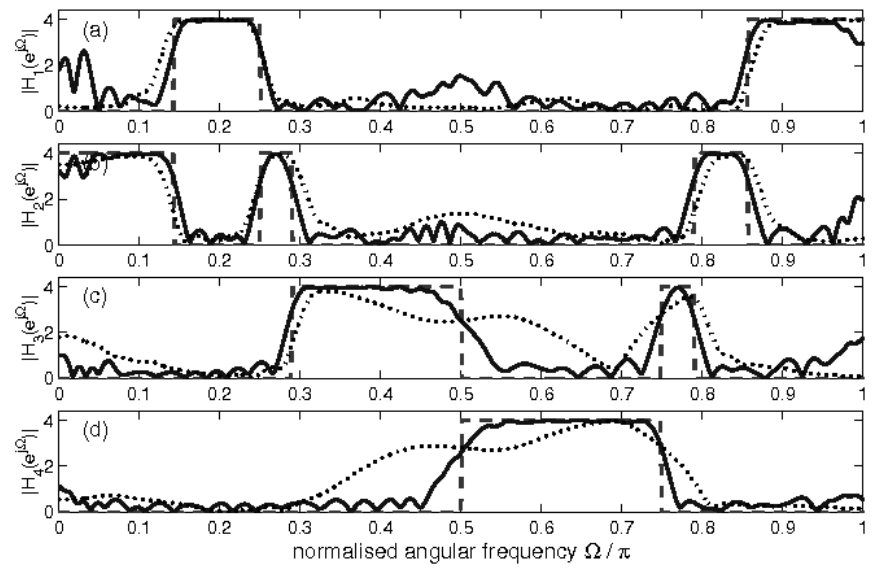

Fig. 3. (a)-(d) magnitude responses of filters $H_{m}(z)$ for theoretical PCFB (dashed), IGA (solid, filter order $N=25$ ) and AEVD (dotted, filter order $N=40$ iterations), obtained for the AR(4) model in Fig. 2(a).

The true PSD of $x[n]$ is shown in Fig. 2(a). Based on this PSD, a theoretical PCFB can be stated, which consists of $M$-band filters with a binary magnitude response and a fragmented passband spectrum in order to yield the theoretical maximum coding gain $G_{\mathrm{PCFB}}$ according to [5], [6]. This theoretical PCFB is shown as a benchmark for various algorithms in Fig. 2(b)-(e) and Fig. 3(a)-(d). The filter banks obtained for SBR2C and SBR2 after $L=150$ iterations are characterised in Fig. 2(b)-(e), and exhibit a close approximation of the PCFB except in spectral regions where the PSD has low values. IGA and AEVD were selected of order $N=25$ and $N=40$ respectively - values which will be justified below in Secs. V-B2 and V-D - yielding the filter banks shown in Fig. 3(a)-(d). The proposed SBR2C algorithm is the closest in approaching the ideal PCFB characteristic. The motivation for the cost function alteration over SBR2 as derived in Sec. III becomes evident when considering the subchannels of weakest power. Fig. 4 underlines this by showing the majorisation properties of both SBR2C and SBR2 after $L=150$ iteration, where 

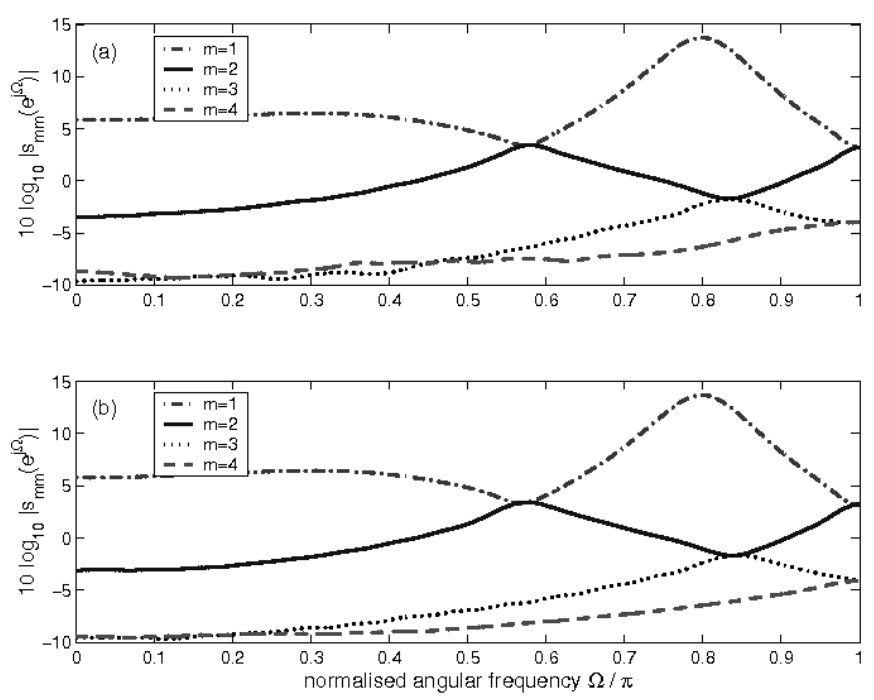

Fig. 4. Spectral majorisation of elements in (14) after $L=150$ iterations for (a) SBR2 and (b) SBR2C, with the latter achieving complete spectral majorisation with $S_{L, 11}\left(e^{j \Omega}\right) \geq \cdots \geq S_{L, 44}\left(e^{j \Omega}\right) \forall \Omega$.

SBR2C fulfills spectal majorisation for all subchannels, while SBR2 does not achieve this for the two weakest subchannels.

2) Coding Gain Evolution : The evolution of coding gain based on the AR(4) process is shown in Fig. 5, comparing the proposed SBR2C algorithm to the benchmarkers. The coding gain results are averaged over different randomised excitations of the AR(4) innovation filter $A(z)$. The upper bound is given by the coding gain, which can be calculated for the theoretical PCFB [5], [6]. For comparison, an $M \times M$ KLT is included, which performs the optimal narrowband decorrelation in the sense of (7). IGA converges quickly with order $N$, but the coding gain value again decreases beyond a value of $N=25$, which had therefore been selected to evaluate the filter banks in Fig. 3 of Sec. V-B1. The IGA algorithm requires an iteration parameter, which gave best results for values of around 1000; however even a substantial increase in this iteration index could not alter the drop in performance. AEVD, SBR2 and SBR2C are based on similar principles, but while AEVD converges quickly, SBR2C achieves a higher coding gain, which, due to its better spectral majorisation performance w.r.t. weaker subbands, also outperforms SBR2.

To verify the result for the AR(4) model, the various algorithms were tested on the randomised MA(14) process for $M=4$ subbands. Since every sample of the MA(14) ensemble has a different associated optimal coding gain $G_{\mathrm{PCFB}}$ as defined by the theoretical PCFB, a normalised coding gain

$$
\hat{G}_{l}^{(\mathrm{n})}=\frac{\hat{G}_{l}}{G_{\mathrm{PCFB}}}
$$

is introduced, which in the case of ideal subband coding converges towards unity. The estimation of the CSD matrix $\mathbf{R}(z)$ or the input PSD $S_{x x}\left(e^{j \Omega}\right)$ is again based on $2^{11}$ samples of $x[n]$. The resulting normalised coding gain averaged over 330 ensemble processes is shown in Fig. 6. The general behaviour of algorithms is similar to the AR(4) system, with

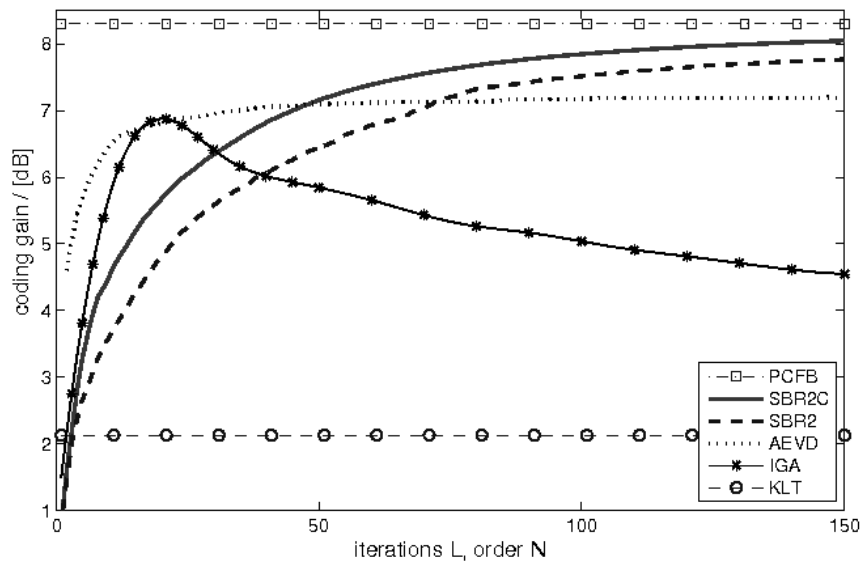

Fig. 5. Evolution of coding gain with iteration number $L$ (SBR2, SBR2C) or filter order $N$ (IGA, AEVD) for AR(4) process.

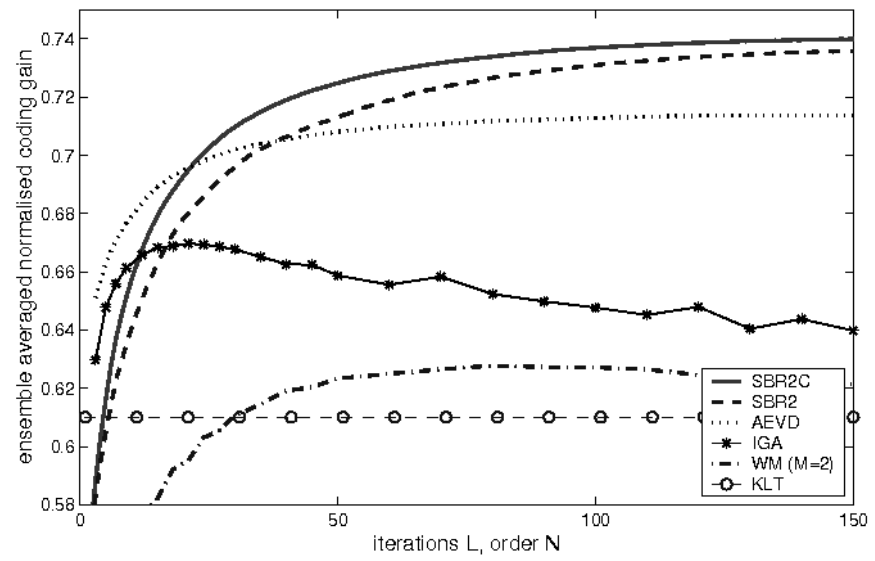

Fig. 6. Evolution of ensemble averaged normalised coding gain with iteration number $L$ (SBR2, SBR2C) or filter order $N$ (IGA, AEVD, WM) for random MA(14) processes. All algorithms operate on $M=4$ subbands, except for WM with $M=2$.

AEVD providing fast initial convergence, and the proposed SBR2C algorithm, achieving the highest normalised coding gain with increasing number of iterations.

The window method (WM) [17] defines a compaction filter for which a complementary filter for an $M=2$ channel filter bank is easily found. Since for values of $M>2$ the method becomes ambiguous, the WM approach is here restricted to the exception $M=2$. While its coding gain reaches a value of around 0.85 when normalised w.r.t. the optimum PCFB coding gain for $M=2$, the increased performance for a PCFB for $M=4$ leads to lower values for $\hat{G}_{l}^{(\mathrm{n})}$ in Fig. 6. While the displayed performance may seem modest, the WM offers computational advantages, as will be seen later.

To underline the impact of the modifications applied in SBR2C over SBR2, Fig. 7 demonstrates the evolution of the compaction gain $\hat{G}_{\text {comp }}$ as defined in (6) and normalised with respect to the optimal performance of the PCFB analogously to (42), analogous considerations for WM as applied in Fig. 6. Since SBR2 gives more weight to the diagonalisation of strong subchannels, its performance w.r.t. compaction gain is superior over the proposed SBR2C algorithm. However, it 


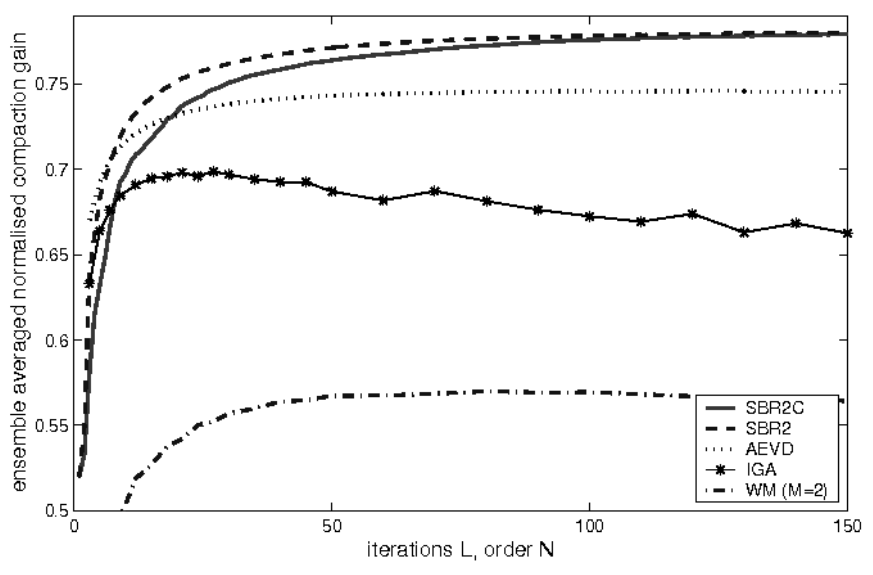

Fig. 7. Evolution of ensemble averaged normalised compcation gain with iteration number $L$ (SBR2, SBR2C) or filter order $N$ (IGA, AEVD, WM) for random MA(14) processes. All algorithms operate on $M=4$ subbands, except for WM with $M=2$. The KLT's average normalised compaction gain of 0.363 is omitted.

is interesting to note that both algorithms converge to very similar compaction gains for an increased number of iterations.

\section{Influence of Estimation Errors}

Sec. IV concentrated on the estimation of the CSD matrix based on data available over a finite window. This section explores the impact of different data window sizes on the accuracy of subband coding algorithms. Given the dominance of SBR2, SRB2C, and AEVD as established on Sec. V-B2, the comparison is restricted to these three methods. As an example, we the utilise the AR(4) innovation filter, with results averaged over 200 different instances of QPSK excitations.

The results for two different window sizes of $x[n], T=2^{11}$ and $T=2^{13}$, are compared with the case of knowledge of true underlying statistics, i.e. $T \rightarrow \infty$. A suitable performance measure is the normalised coding gain error,

$$
\hat{E}_{l}^{(n)}=\frac{G_{\mathrm{PCFB}}-\hat{G}_{l}}{G_{\mathrm{PCFB}}}=1-\hat{G}_{l}^{(n)},
$$

which assesses the normalised mismatch w.r.t. the performance of the ideal PCFB. For an optimal algorithm, $\hat{E}_{l}^{(n)}$ is expected to converge to zero for large $T$ and sufficient iterations $L$ in case of SBR2 and SBR2C or filter order $N$ in case of the AEVD. The results in Fig. 8 show the superiority of SBR2C over SBR2 in terms of convergence to the PCFB performance. The graph also highlights the performance difference between the suboptimal and optimal estimation methods for $\hat{\mathbf{R}}(z)$ described in Sec. IV-A, which leads to an alteration in the effective data size by a factor of $M=4$. The results for the next best competitor to SBR2C, the AEVD, are only shown for the knowledge of true statistics, which is still outperformed by SBR2 for a sufficiently high number of iterations.

\section{Computational Complexity}

1) Iterations, Filter Order, and Run Time Complexity: The number of iterations $L$, which governs the convergence

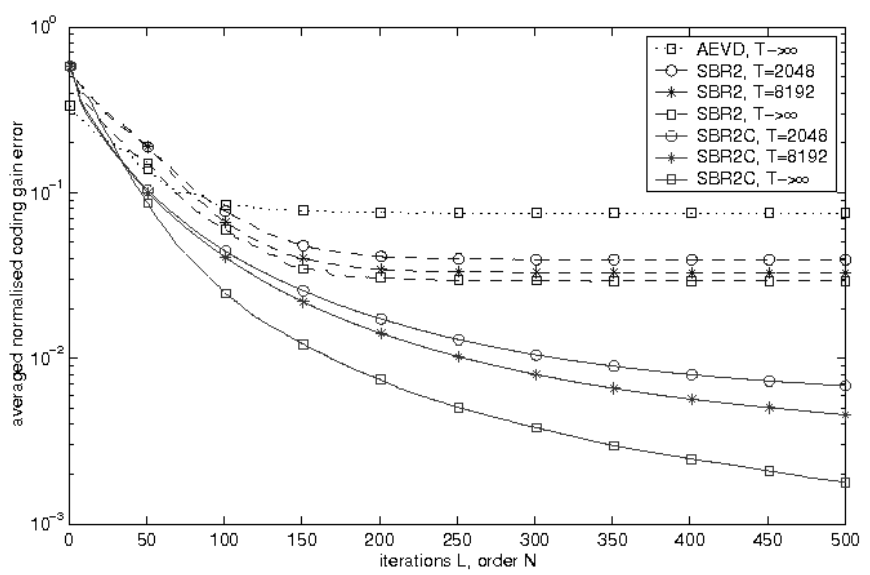

Fig. 8. Normalised coding gain error obtained for AR(4) process with $M=4$ with SBR2C (solid), SBR2 (dashed), and AEVD (dotted) for knowledge of true statistics (square), and finite data windows of $x[n]$ with $T=8192$ (star) and $T=2048$ (circle); for the latter two, curves are averaged over an ensemble of 50 variations on the excitation sequence of the innovation filter $A(z)$.

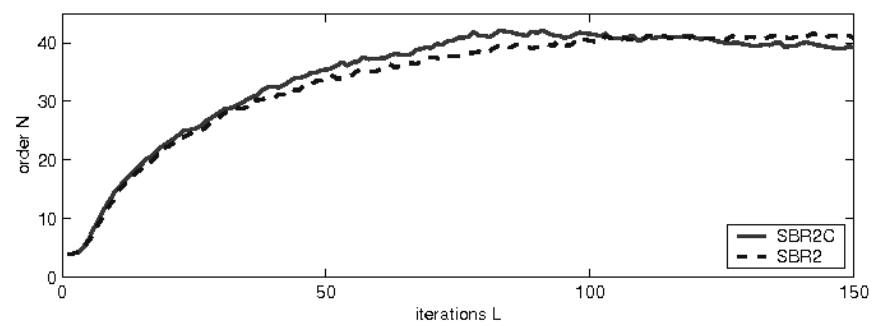

Fig. 9. Dependency of filter order $N$ on iteration number $L$ for SBR2 and SBR2C.

of SBR2 and SBR2C, and the filter order $N$, which is the main parameter of the remaining benchmark methods, have been shown together in previous plots but are not directly equivalent. With order trimming during the iteration process applied to both SBR2 and SBR2C as mentioned in Sec. II-E and outlined in [22], the relation between $N$ and $L$ has been measured for the randomised MA(14) process. The averaged results on the dependency between $N$ and $L$ are given in Fig. 9, showing an initial fast growth in order $N$ and a gradual slowing and saturation as iterations $L$ progress. Although measured for MA(14), these results have been found for all systems attempted, and motivate the selection of $L=150$ and $N=40$ in Figs. 2 and 3 in Sec. V-B1 for SBR2/SBR2C and AEVD, respectively, to yield systems of comparable order.

The run time complexity defines the computation cost of applying the PU encoder matrix $\mathbf{H}(z)$ to input data, and is hence directly related to the filter order $N$. Therefore, the relation between the achievable coding gain and run time complexity is similar to the results displayed in Fig. 6, whereby the curves for SBR2 and SBR2C are non-linearly compressed according to Fig. 9 into the range $N \approx[1,40]$, increasing both the superiority of the AEVD at low orders $(N<25)$, and the superiority of SBR2C at moderate to high orders $(N>25)$.

2) Algorithm Complexity: The complexity of determining the encoder matrix $\mathbf{H}(z)$ based on the various algorithms 


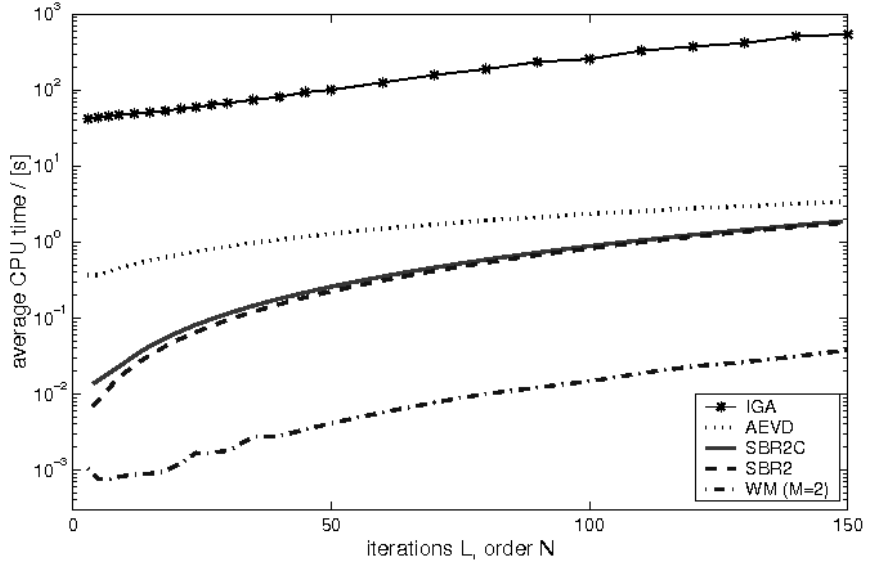

Fig. 10. CPU time requirement for calculation of encoder matrix $\mathbf{H}(z)$ for different approaches as a function of iteration number $L$ (SBR2 and SBR2C) or filter order $N$ (IGA, AEVD, and WM).

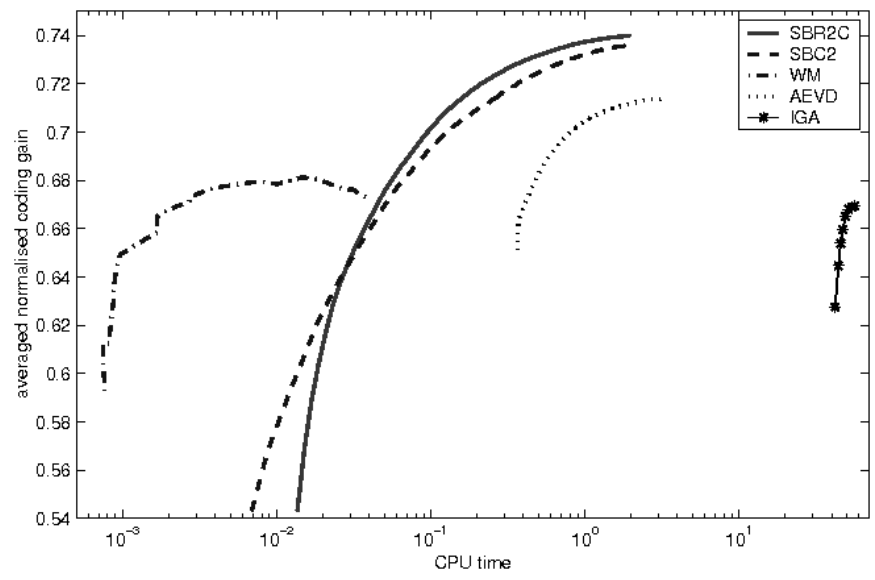

Fig. 11. Achievable normalised coding gain versus $\mathrm{CPU}$ time required to calculate the encoder matrix $\mathbf{H}(z)$.

has been assess by the CPU time measured during $400 \mathrm{~h}$ of simulations for the coding gain evolution across an ensemble of 330 randomised $\mathrm{MA}(14)$ processes. Fig. 10 reveals the IGA as a very complex algorithm, followed by the AEVD. The overhead of normalising the cross-correlation functions in SBR2C results in a small complexity degradation w.r.t. SBR2 particularly at lower orders, while WM. although restricted to $M=2$, represents an approach with very low complexity compared to IGA, AEVD, and SBR2/SBR2C.

To assess the achievable coding gain in terms of the effort of calculating the encoder matrix, Fig. 11 relates the results of Fig. 10 to Fig. 6 for the randomised MA(14) processes. At low complexity, the window method can deliver the best coding gain. Otherwise, the SBC2C algorithm offers the best coding performance at the lowest cost compared to SBR2, AEVD, and IGA.

\section{Discussion AND CONCLUSIONS}

In this paper, we have investigated paraunitary filter bank design techniques for subband coding. Specifically, we have related principal component filter banks to a polynomial matrix EVD, which is applied to the CSD matrix of the polyphase component vector of the signal to be encoded. Spectral majorisation is a key condition for good coding gain performance, which has motivated the extension of an existing PEVD algorithm, the SBR2 algorithm in [22] by an alternative cost function, leading to the SBR2 algorithm for subband coding, or SBR2C. Since optimal estimation of the CSD matrix is crucial, two estimation techniques have been considered, which exploit the implicit structure of the CSD matrix, possessing both Parahermitian and pseudo-circulant properties. Compared to a direct computation, the optimal approaches are equivalent to effectively increasing the data window by a factor $M$, when an $M$-channel subband coder is considered.

The proposed SBR2C algorithm can converge to a solution that yields an $M$-channel perfect reconstruction filter bank, which is approximately optimal for subband coding in a small number of iterations. The solution provided by the PEVD obtained by SBR2C converges to that provided by the principle component filter bank (PCFB) for subband signals as the number of iterations increases. The coding performance of the proposed algorithm has been demonstrated to exceed current state-of-the-art methods, such as the iterative greedy algorithm (IGA), the approximate EVD (AEVD), or the standard SBR2 algorithm for moderate to large filter orders on a set of benchmark problems. When considering the calculation of the encoder matrix, the window method has been shown to yield moderate coding gain performance at a very low cost. On the same benchmark problems, when admitting moderate to high filter order, the SBR2C algorithm provides the best coding performance at the lowest cost.

The proposed SBR2C algorithm has been mainly targetted towards subband coding, since a number of competitor algorithms and benchmarks exist in the area. However, since the algorithm applies a PEVD to the CSD matrix rather than analysing the PSD of the single-channel input signal, the applicability of the derived SBR2C algorithm is considerably wider, and can include multi-channel coding or MIMO subband coding.

\section{APPENDIX A \\ Pseudo-Circulant Property of $\mathbf{R}(z)$.}

In the following, we show that the cross-spectral density matrix $\mathbf{R}(z)$ of the demultiplexer output $\mathbf{x}[k]$ in Fig. 1(b) is a pseudo-circulant matrix for a WSS input signal. The crosscorrelation between the $m$ th and $p$ th polyphase components, $r_{m, p}[\tau]$, can be - according to the description in (30) expressed in terms of the input signal $x[n]$ to the demultiplexer and its auto-correlation $r[\tau]=\mathcal{E}\left\{x[n] x^{*}[n-\tau]\right\}$,

$$
\begin{aligned}
r_{m, p}[\tau] & =\mathcal{E}\left\{x_{m}[k] x_{p}^{*}[k-\tau]\right\} \\
& =\mathcal{E}\left\{x[M k-m+1] x^{*}[M k-p+1-M \tau]\right\}(44) \\
& =r[M \tau+p-m] .
\end{aligned}
$$

The step from (44) to (45) is based on the wide sense stationarity of $x[n]$, which retains this property when decimated to $x[M n]$ [12]. From (45), an entry of the CSD matrix $\mathbf{R}(z)$ in 
(32) can be denoted as

$$
r_{m, p}(z)=\sum_{\tau=-\infty}^{\infty} r[M \tau+p-m] z^{-\tau} .
$$

With the polyphase decomposition of the auto-correlation sequence

$$
r(z)=\sum_{m=0}^{M-1} \phi_{m}\left(z^{M}\right) z^{-m}
$$

we need to distinguish two cases in order to identify (46) with one of the polyphase components $\phi_{m}(z) \bullet \longrightarrow \phi_{m}[\tau]=$ $r[M \tau+m]$ :

a) Case $p \geq m$ : Since $0 \leq p-m<M$,

$$
r_{m, p}(z)=\phi_{p-m}(z) \text {. }
$$

b) Case $p<m$ : Therefore $0 \leq M+p-m<M$, and the insertion of a spurious $M$ into the argument of the auto-correlation sequence leads to

$$
\begin{aligned}
r_{m, p}(z) & =\sum_{\tau=-\infty}^{\infty} r[M(\tau-1)+M+p-m] z^{-\tau} \\
& =\sum_{\nu=-\infty}^{\infty} r[M \nu+M+p-m] z^{-\nu-1} \\
& =z^{-1} \phi_{M+p-m}(z) .
\end{aligned}
$$

With the substitution $\nu=\tau-1$ in (49), the two cases (48) and (50) confirm the pseudo-circulant property of $\mathbf{R}(z)$ as defined in (34) and (35).

\section{REFERENCES}

[1] S. Akkarakaran and P.P. Vaidyanathan, "On optimization of filter banks with denoising applications," in IEEE International Symposium on Circuits and Systems, July 1999, vol. 3, pp. 512-515.

[2] P. Desarte, B. Macq, and D.T.M. Slock, "Signal-adapted multiresolution transform for image coding," IEEE Transactions on Information Theory, vol. 38, no. 2, pp. 897-904, March 1992.

[3] P.P. Vaidyanathan, "Filter banks in digital communications," IEEE Circuits and Systems Magazine, vol. 1, no. 2, pp. 4-25, Apr. 2001.

[4] L. Vandendorpe, L. Cuvelier, F. Deryck, J. Louveaux, and O. van de Wiel, "Fractionally spaced linear and decision-feedback detectors for transmultiplexers," IEEE Transactions on Signal Processing, vol. 46, no. 4, pp. 996-1011, Apr. 1998.

[5] M.K. Tsatsanis and G.B. Giannakis, "Principal component filter banks for optimal multiresolution analysis," IEEE Transactions on Signal Processing, vol. 43, no. 8, pp. 1766-1777, Aug. 1995.

[6] M. Unser, "On the optimality of ideal filters for pyramid and wavelet signal approximation," IEEE Transactions on Signal Processing, vol. 41, no. 12, pp. 3591-3596, Dec. 1993.

[7] S. Akkarakaran and P.P. Vaidyanathan, "Filterbank optimization with convex objectives and the optimality of principal component forms," IEEE Transactions on Signal Processing, vol. 49, no. 1, pp. 100-114, January 2001.

[8] P.P. Vaidyanathan, "Theory of optimal orthonormal subband coders," IEEE Transactions on Signal Processing, vol. 46, no. 6, pp. 1528-1543, June 1998.

[9] Simon Haykin, Adaptive Filter Theory, Prentice Hall, 4th edition, 2002.

[10] G. H. Golub and C. F. Van Loan, Matrix Computations, John Hopkins University Press, Baltimore, Maryland, 3rd edition, 1996.

[11] J. Huang and P. Schultheiss, "Block quantization of correlated gaussian random variables," IEEE Transactions on Communications, vol. 11, no. 3, pp. 289-296, September 1963.

[12] P. P. Vaidyanathan, Multirate Systems and Filter Banks, Prentice Hall, Englewood Cliffs, 1993.

[13] A. Kirac and P.P. Vaidyanathan, "On Existence of FIR Principal Component Filter Banks," in IEEE International Conference on Acoustics, Speech and Signal Processing, May 1998, vol. 3, pp. 1329-1332.
[14] H. Caglar, Y. Liu, and A. N. Akansu, "Statistically Optimized PR-QMF Design," in Proc. SPIE 1605, Wavelet Appl. Signal Image Processing, San Diego, CA, 1991, pp. 86-94.

[15] B. Xuan and R.I. Bamberger, "FIR Principal Component Filter Banks," IEEE Transactions on Signal Processing, vol. 46, no. 4, pp. 930-940, Apr. 1998.

[16] P. Moulin and M.K. Mihcak, "Theory and Design of Signal-Adapted FIR Paraunitary Filter Banks," IEEE Transactions on Signal Processing, vol. 46, no. 4, pp. 920-929, Apr. 1998.

[17] A. Kirac and P.P. Vaidyanathan, "Theory and Design of Optimum FIR Compaction Filters," IEEE Transactions on Signal Processing, vol. 46, no. 4, pp. 903-919, April 1998.

[18] J. Tuqan and P.P. Vaidyanathan, "A State Space Approach to the Design of Globally Optimal FIR Energy Compaction Filters," IEEE Transactions on Signal Processing, vol. 48, no. 10, pp. 2822-2838, Oct. 2000.

[19] A. Tkacenko and P.P. Vaidyanathan, "On the Spectral Factor Ambiguity of FIR Energy Compaction Filter Banks," IEEE Transactions on Signal Processing, vol. 54, no. 1, pp. 380-385, Jan. 2006.

[20] A. Tkacenko and P.P. Vaidyanathan, "Iterative Greedy Algorithm for Solving the FIR Paraunitary Approximation Problem," IEEE Transactions on Signal Processing, vol. 54, no. 1, pp. 146-160, Jan. 2006.

[21] J. G. McWhirter and P. D. Baxter, "A Novel Technqiue for Broadband SVD," in 12th Annual Workshop on Adaptive Sensor Array Processing, MIT Lincoln Labs, Cambridge, MA, 2004.

[22] J. G. McWhirter, P. D. Baxter, T. Cooper, S. Redif, and J. Foster, "An EVD Algorithm for Para-Hermitian Polynomial Matrices," IEEE Transactions on Signal Processing, vol. 55, no. 5, pp. 2158-2169, May 2007.

[23] J.A. Foster, J.G. McWhirter, M.R. Davies, and J.A. Chambers, "An Algorithm for Calculating the QR and Singular Value Decompositions of Polynomial Matrices," IEEE Transactions on Signal Processing, vol. 58, no. 3, pp. 1263-1274, March 2010.

[24] A. Tkacenko, "Approximate Eigenvalue Decomposition of ParaHermitian Systems Through Successive FIR Paraunitary Transformations," in IEEE International Conference on Acoustics Speech and Signal Processing, Mar. 2010, pp. 4074-4077.

[25] P.A. Regalia and P. Loubaton, "Rational Subspace Estimation Using Adaptive Lossless Filters," IEEE Transactions on Signal Processing, vol. 40, no. 10, pp. 2392-2405, Oct. 1992.

[26] P.A. Regalia and Dong-Yan Huang, "Attainable Error Bounds in Multirate Adaptive Lossless FIR Filters," in IEEE International Conference on Acoustics, Speech, and Signal Processing, May 1995, vol. 2, pp. $1460-1463$

[27] R. H. Lambert, M. Joho, and H. Mathis, "Polynomial Singular Values for Number of Wideband Source Estimation and Principal Components Analysis," in Proc. Int. Conf. Independent Component Analysis, 2001, pp. 379-383.

[28] S. Redif and T. Cooper, "Paraunitary Filter Bank Design via a Polynomial Singular-Value Decomposition," in IEEE International Conference on Acoustics, Speech, and Signal Processing, 2005, vol. 4, pp. 613-616.

[29] T. Kailath, Linear Systems, Prentice Hall, 1980.

[30] S. Redif, J. G. McWhirter, and S. Weiss, "Orthonormal Subband Coder Design Using Polynomial Eigenvalue Decomposition," in 18th European Signal Processing Conference, Aalborg, Denmark, August 2010, pp. 437-441.

[31] A. Papoulis, Probability, Random Variables, and Stochastic Processes, McGraw-Hill, New York, 3rd edition, 1991.

[32] I. Tabus, C. Popeea, and J. Astola, "A Design Procedure for Optimal Energy Compaction IIR Filters," IEEE Transactions on Circuits and Systems II: Analog and Digital Signal Processing, vol. 48, no. 7, pp. 740-744, July 2001.

[33] B. Mulgrew, P. Grant, and J. Thompson, Digital Signal Processing: Concepts and Applications, Palgrave Macmillan, 1999.

[34] Y.-P. Lin and S.-M. Phoong, "Smith form of FIR Pseudo-Circulants," IEEE Signal Processing Letters, vol. 9, no. 8, pp. 256-258, August 2002.

[35] O.S. Jahromi, M.A. Masnadi-Shirazi, and M. Fu, "A Fast $\mathcal{O}(n)$ Algorithm for Adaptive Filter Bank Design," in IEEE International Conference on Acoustics, Speech and Signal Processing, Ma 1998, vol. 3, pp. $1325-1328$. 


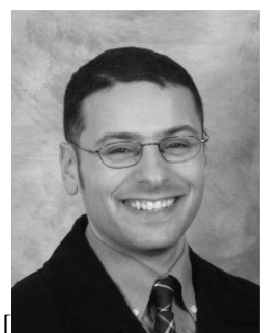

Soydan Redif received the B.Eng. degree (first class hons.) in Electronic Engineering from Middlesex University, London, in 1998, and the Ph.D. degree from the University of Southampton in 2006.

In 1999, he joined the Defence, Evaluation and Research Agency, working initially on airborne SHF SATCOM systems. From 2000 until 2007, he was with the Advanced Signal and Information Processing group at QinetiQ, Malvern, U.K., where he carried out research on adaptive signal processing. From 2008 to 2011, he was an Assistant Professor in the Electrical and Electronic Department at Near East University (NEU), Nicosia, Cyprus. During 2011, he was a Visiting Lecturer at the European University of Lefke (EUL), Lefke, Cyprus. He is currently an Assistant Professor with the Electrical and Electronic Department at EUL. His main research interests are in adaptive multirate signal processing, broadband sensor arrays, convolutive blind signal separation, and polynomial matrix techniques. In particular, he has worked on the design of algorithms for data compression, interference rejection, and the detection and localisation of broadband signals.

Dr Redif is a Chartered Engineer, a Member of the IET, a Member of the IEEE, and a Member of EURASIP. He was the recipient of the IEE Award for outstanding academic achievement in 1998.

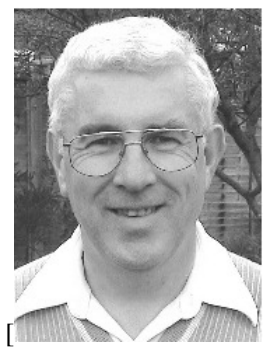

John G. McWhirter received the first class honors degree in mathematics and the Ph.D. degree in theoretical physics from the Queens University of Belfast, Belfast, Ireland, in 1970 and 1973, respectively.

He joined the Royal Radar Establishment in Malvern (later to become the Royal Signals and Radar Establishment, and now part of QinetiQ Ltd.) in 1973, where he became a Senior Fellow in the Centre for Signal and Information Processing Group. In 2007, he left QinetiQ to take up his current post as Distinguished Research Professor in the Engineering Department, Cardiff University, Cardiff, U.K. He is also a Visiting Professor in Electrical Engineering at the Queens University of Belfast. He has been carrying out research on adaptive signal processing since 1980 and was awarded the J. J. Thomson Medal by the Institution of Electrical Engineers in 1994 for his research on systolic arrays. He has published more than 140 research papers and holds numerous patents. His current research is devoted to broadband sensor arrays, convolutive blind signal separation, and polynomial matrix techniques. The signal processing group which he built up in Malvern over many years, received the EURASIP Group Technical Achievement Award for 2003.

Dr. McWhirter was elected as a Fellow of the Royal Academy of Engineering in 1996 and as a Fellow of the Royal Society in 1999. He is a Fellow of the Institute of Mathematics and its Applications (IMA) and served as President of the IMA in 2002 and 2003. He is also a Fellow of the Institute of Electrical Engineers, a Fellow of the Institute of Physics, and a member of the London Mathematical Society.

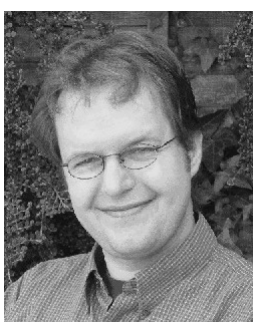

Stephan Weiss received a Dipl.-Ing. degree from the University of Erlangen-Nürnberg, Erlangen, Germany, in 1995, and a Ph.D. degree from the University of Strathclyde, Glasgow, Scotland, in 1998, both in electronic and electrical engineering.

$\mathrm{He}$ is currently a Reader in the Department of EEE at Strathclyde. From 1999 until 2006, he held lecturer and senior lecturer positions within the School of Electronics and Computer Science at the University of Southampton. Prior to this, he was a Visiting Lecturer at the University of Strathclyde in 1998/99 and a Visiting Scholar at the University of Southern California in 1996/97. His research interests lie in adaptive, multirate, and array signal processing with applications in communications, audio, and biomedical signal processing, where he has published more than 180 technical papers. For his work in biomedical signal processing, he was a co-recipient of the 2001 research award of the German society on hearing aids. In 2011 he was a corecipient of the VTC-Spring best paper award in the MIMO systems track.

Dr Weiss is a member of EURASIP and a senior member of the IEEE. He was the technical co-chair for EUSIPCO 2009 in Glasgow, and an associate editor for Elsevier Signal Processing and IEEE Transactions on Mobile Computing. 